\title{
Verily and Its Approach to Digital Biomarkers
}

\author{
Interview with Dr. William J. Marks, Jr., Head of Clinical Neurology at \\ Verily Life Sciences
}

Digital Biomarkers: This is Ray Dorsey, the editor of Digital Biomarkers. Today, I'm very pleased to be joined by Dr. William Marks, who is the Head of Clinical Neurology for Verily. Bill, thanks very much for joining us.

William Marks: You're welcome.

Digital Biomarkers: Bill, I was hoping you could first tell us who or what is Verily?

William Marks: Verily is a translational life sciences company, living at the intersection of technology, data science, and healthcare. Formerly called Google Life Sciences, we're now an independent company that's part of the Alphabet organization. Our mission is to make the world's health data useful so that people enjoy healthier lives. We strive to collect and integrate a

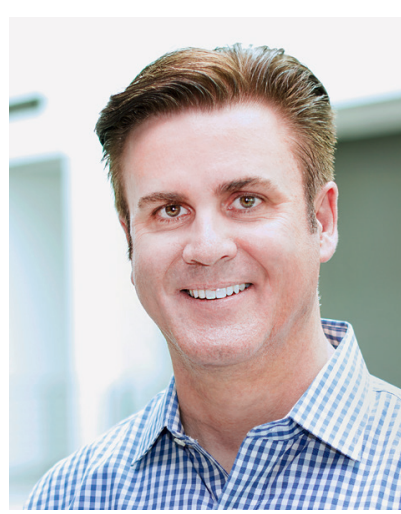

William J. Marks, Jr. broad spectrum of existing and new types of data around health and disease to reveal informative and actionable insights. We think a lot about health and disease, and the transition between the two. Our ultimate goal is to enable earlier and more precise diagnoses, monitoring of progressive diseases with better sensitivity, and - ultimately - care delivery in a more efficient, effective, and proactive manner.

Verily has brought together basic scientists, software engineers, hardware engineers, clinicians, clinical researchers, and those from many other disciplines - all under one roof, working on tackling some of the toughest issues in healthcare. We feel that it's only through this cross-disciplinary approach that we'll be able to obtain important answers to very challenging questions. Some organizations focus mostly on software analytics, others on sensors and other hardware, or still others on molecular assays; we at Verily are bringing all of these capabilities together, and more. 
Digital Biomarkers: Before joining Verily, you were a Professor of Neurology at the University of California, San Francisco, where you were for nearly 20 years, and you are one of the leading authorities on neuromodulation for Parkinson's disease and epilepsy. What made you leave academia for Verily?

William Marks: The reason that I shifted environments was my realization that the big breakthroughs in medicine will likely require the approach that Verily is embracing. The next advances in healthcare will require the development of new data collection tools, powerful computing infrastructure, thoughtful and novel analytic capabilities, and machine-learning expertise. There needs to be an immense infrastructure to do that well. For me, being at Verily is an amazing opportunity to contribute to the development and application of these platforms to ultimately care for individuals and populations, in health and in disease.

Digital Biomarkers: Verily has launched projects in cardiovascular disease, multiple sclerosis, and Parkinson's disease, among others, and it's very active in "All of Us," the Precision Medicine Initiative. Could you tell us a little bit about one or two of these projects?

William Marks: Verily's goal is to target some of the most vexing problems and to make a major impact in bringing new solutions. We focus on health issues that are extremely common, like diabetes or cardiovascular disease, and on those that are extremely challenging, like progressive neurological disorders.

These are exemplar conditions that lend themselves to both deep and broad phenotyping. To understand such complex conditions better, we believe it is important to study them in a multi-dimensional manner, from molecular to macroscopic, and then integrate these varied data streams to arrive at a more reliable picture of what's going on.

Digital Biomarkers: I was recently in Silicon Valley and a colleague remarked that he thinks over the next decade Verily is going to be the largest healthcare company in the world. Do you have any thoughts on that?

William Marks: We have a lot of ambitious goals here, and what has really been heartening to me, Ray, is the long-term perspective that the leadership team of the company, including our CEO, Andrew Conrad, has emphasized. We first need to build key tools and capabilities to gather informative types of data. We need to build analytic platforms to make meaningful sense of these data. We understand that this is a long-term proposition, but with that point of view we are poised to successfully build and assemble the critical components - like the tools, analytic expertise, computing power, and machine-learning capabilities - all with guidance from clinicians and scientists. With all of these elements thoughtfully brought together, I believe we have a great opportunity to make major contributions to advance human health.

Digital Biomarkers: On human health, Parkinson's disease, like many neurodegenerative conditions, is really limited by its reliance on subjective, likely insensitive, and episodic outcome measures that could only be performed in the clinic. What are we missing for Parkinson's disease?

William Marks: Well, you've identified a key issue, Ray. As we all know, in so many of our clinical studies we're trying to ascertain whether an intervention is decelerating disease progression or even effectively treating symptoms. We rely on cross-sectional measures that are obtained very briefly in the artificial setting of a clinic visit or during the even more rarified clinical study visit. And of course we know that so much more goes on throughout the course of patients' daily lives.

I think Parkinson's disease is a great example of where we have an opportunity to obtain a deeper and more real-world assessment of patients' function. Currently, with all the clinical variability from patient to patient, and with the imperfect scoring systems and clinical tools available, we have a limited snapshot of information upon which to draw conclusions or take action. 
I suspect we are missing important signals into understanding diseases using the limited tools that have been available to us - so that's why I'm really excited about the recent availability of new sources of data, including data from devices that may provide digital biomarkers to obtain longer-term representations of function during the usual daily lives of patients. We are engaged in multiple efforts to collect these types of data, taking us outside the four walls of the clinic so that we can identify new markers of disease progression, or signals that could help clinicians with treatment decisions.

An example of a tool we are using for this is the Verily Study Watch, an investigational device we developed for medical research that unobtrusively collects physiological and environmental data from individuals over long periods. Data from these types of devices could go a long way to establishing outcomes for studies that have more relevance to patients, rather than some of the subjective approaches that we currently use to categorize whether somebody's responding to a treatment or not. For Parkinson's, in particular, we have a real unmet need that we can now begin to fill with the advent of these technological advances.

It seems likely, though, that even a sophisticated wearable system coupled with advanced data analytics providing near-continuous, large-scale data sets about function will not sufficiently characterize all aspects of function, symptomatology, or disease stage. These data need to be thoughtfully integrated with other data types, like imaging, patient reports of function, genetic markers, molecular phenotyping, and so on. It's bringing together data in this multidimensional approach that has the promise to allow us to understand complex diseases like Parkinson's disease in new ways and characterize these conditions better. With this will come new endpoints for studies that should accelerate the development of symptomatic and disease-modifying treatments. Ultimately, the payoff of this integrative data approach should be tools to guide patients and clinicians in managing disease in a more personalized, precise, and effective manner.

Digital Biomarkers: To help fill in this gap in Parkinson's disease, Verily has recently announced a partnership with Radboud University Medical Center and ParkinsonNet in the Netherlands to conduct molecular analysis, brain imaging, and assessments with wearable devices on about 650 individuals with Parkinson's disease in the Netherlands. What do you hope to find from this kind of study?

William Marks: The collaboration between Verily, Professor Bastiaan Bloem and colleagues at Radboud University, and ParkinsonNet is something that I'm extremely excited about. We're tapping into the ParkinsonNet system in the Netherlands, where nearly everyone with Parkinson's disease is included in a national care network, to recruit subjects for the study. In the study, called the Personalized Parkinson Project, we are focusing on patients diagnosed within the past 5 years who are more likely to be in the earlier, milder stages of the disease. We'll follow these patients, as they wear the Verily Study Watch, and look broadly and deeply at any array of data to understand the disease, its phenotypes, and its progression in entirely new ways.

Of course, there are other cohort studies in Parkinson's disease, and we intend to build upon those. We've harmonized our approaches with other studies, wherever possible, and then augmented with the newest capabilities that we have. As new technologies become available, we plan to implement those in the study as well. We intend to bring to the scientific community a rich data repository for analysis.

Digital Biomarkers: Can you discuss a little bit more your novel approach to data sharing and privacy? And then, can you also address the, maybe sometimes unspoken, concern that a for-profit company, like Verily or Alphabet, is gathering data, and sometimes sensitive data, on a number of individuals pertaining to the health and how these data may or may not be used in the future. 
William Marks: It's incredibly important that in this new age of collecting so much information from individuals in their normal daily lives that we all incorporate stringent data privacy protections and security measures so that we can maintain trust among those participating in research or clinical care. We're very serious about data protection at Verily. As we embarked on our partnership with Radboud University in the Netherlands, a country that emphasizes privacy and protection of personal information, one of the attractive features of this collaboration was working with the Radboud University Digital Security Group.

That team at Radboud, led by Professor Bart Jacobs, has developed a security methodology called PEP, which stands for Polymorphic Encryption and Pseudonymization. It's a very sophisticated architecture for collecting, pseudonymizing, storing, and then ultimately analyzing data. Investing in protocols that improve and enhance security and privacy will not only help us for this specific study but also help the research community in general with data sharing across studies, institutions, and geographic boundaries.

Digital Biomarkers: Great. Bill, you are very kind to be willing to devote your time to serve on the advisory board of our new journal, Digital Biomarkers, which is seeking to be a journal and a community aimed at fostering the development of these new digital tools to improve health. What are your hopes for this new journal and the community?

William Marks: It's so exciting to me to hear that the journal is being developed and, in particular, that you've taken on the editorship, Ray. Digital biomarker technology has moved ahead very rapidly. There's a lot of work in this area going on in a range of areas, largely in silos. Certainly, this work crosses so many disciplines, and there's a real need for a venue that that can bring together those from different backgrounds and disciplines and with different types of expertise who all care about similar outcomes.

This approach has certainly been a central tenet of Verily: bringing together people from different backgrounds to work as a team to tackle tough problems. In many ways, the journal will bring together people from a lot of different backgrounds and highlight the work that is going on in these areas so that people can learn more rapidly from each other. I think we have a real opportunity to traverse many disease areas with digital phenotyping. This will help the field accelerate the capabilities that will be available across clinical research and medicine.

Digital Biomarkers: Thanks very much. If you had to think about the most important question that this community should address, what would that be?

William Marks: How can we validate the signals that we're receiving from these digital biomarker approaches with ground truth? "Ground truth" is a phrase used a lot at Verily. We're starting to think about how we monitor the human body to understand health and disease, and to apply very sophisticated machine learning to the data streams that we're acquiring. Yet how and when do we know that the signals we are extracting are important, biologically relevant, clinically significant, useful to the patient, or meaningful to ultimate outcomes? This is a complex endeavor when so many of our existing standards are quite imperfect. It just seems to me that one of the fundamental foundational issues with which we need to grapple is understanding and validating the clinical relevance of data from these new technologies. 